\title{
Application Article \\ Decoupling of Multifrequency Dipole Antenna Arrays for Microwave Imaging Applications
}

\author{
E. Saenz, ${ }^{1}$ K. Guven, ${ }^{2,3}$ E. Ozbay, ${ }^{2}$ I. Ederra, ${ }^{1}$ and R. Gonzalo ${ }^{1}$ \\ ${ }^{1}$ Electrical and Electronic Engineering Department, Public University of Navarra, Campus Arrosadia, 31006 Pamplona, Spain \\ ${ }^{2}$ Nanotechnology Research Center, Bilkent University, Bilkent, Ankara, 06800, Turkey \\ ${ }^{3}$ Department of Physics, Koc University, Istanbul 34450, Turkey
}

Correspondence should be addressed to E. Saenz, elena.saenz@unavarra.es

Received 6 September 2009; Accepted 25 November 2009

Academic Editor: Hoi Shun Lui

Copyright (C) 2010 E. Saenz et al. This is an open access article distributed under the Creative Commons Attribution License, which permits unrestricted use, distribution, and reproduction in any medium, provided the original work is properly cited.

\begin{abstract}
The mutual coupling between elements of a multifrequency dipole antenna array is experimentally investigated by $S$-parameter measurements and planar near-field scanning of the radiated field. A multifrequency array with six dipoles is analyzed. In order to reduce the coupling between dipoles, a planar metasurface is placed atop the array acting as superstrate. The mutual coupling of the antenna elements in the absence and presence of the superstrate is presented comparatively. Between 3 and $20 \mathrm{~dB}$ mutual coupling reduction is achieved when the superstrate is used. By scanning the field radiated by the antennas and far-field measurements of the radiation pattern, it is observed that the superstrate confines the radiated power, increases the boresight radiation, and reduces the endfire radiation.
\end{abstract}

\section{Introduction}

Microwave imaging is a wide field that covers all processes in which measurements of electromagnetic fields in the microwave region are used for creating images $[1,2]$. Nowadays, new applications are arising in aviation, the automotive industry, astronomy, earth observation, medicine, and security. To create images from microwave measurements a microwave camera able to transmit microwaves and measure the scattered waves at one or more antennas is necessary. In order to improve the resolution, measurements at different frequencies of illuminating electromagnetic field can be performed, since reconstruction algorithms can achieve better accuracy using these multifrequency (MF) data [2].

By means of lithographic techniques, large twodimensional planar receivers (single or MF arrays) can be easily manufactured, reducing the cost and simplifying the assembly process with respect to nonplanar techniques. Each element of the antenna array will be a pixel in the camera and, therefore, they must be isolated. Waveguide/feedhorn technology provides a natural isolation between adjoining pixels. In contrast, one of the main disadvantages of planar arrays is that they suffer from coupling between elements, which in most cases results in a degradation of the expected radiation features.

In a planar dielectric substrate the parasitic crosscoupling is mainly via surface modes, being more significant when high dielectric constant materials or thick substrates are used. Consequently, electromagnetic bandgap (EBG) structures, which prevent the propagation of radiation for frequencies within the bandgap, have attracted much interest and proven their effectiveness as surface wave suppressing substrates $[3,4]$. Conversely, when low dielectric substrates are used, air coupling is the dominant coupling mechanism. In order to reduce this mutual coupling, improving at the same time the radiation performance of the antennas, metasurfaces as a superstrate of the antennas have been proposed by the authors. In [5], a nonplanar metasurface was used as superstrate of a single dipole and an array of dipoles. In order to reduce the thickness of the configuration, a new planar metasurface was designed afterwards [6]. Measurements of the effect of this metasurface in the radiation properties of a dipole antenna were reported in [7]. Then, an array configuration formed by $2 \times 2$ dipoles with the proposed metasurface was designed. Planar near-field measurements of the dipole and the array were presented in [8]. Finally, 


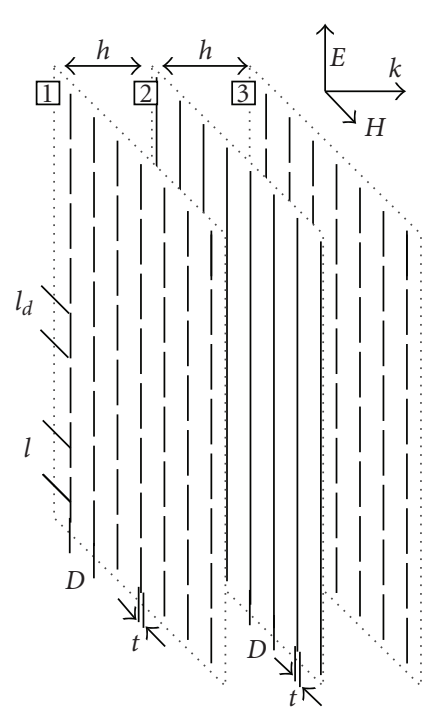

(a)

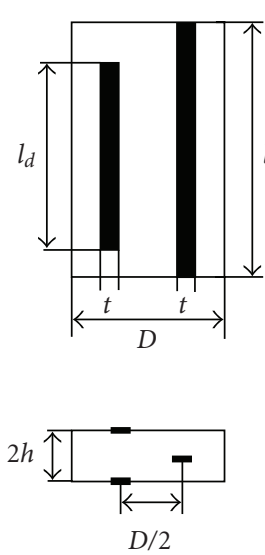

(b)

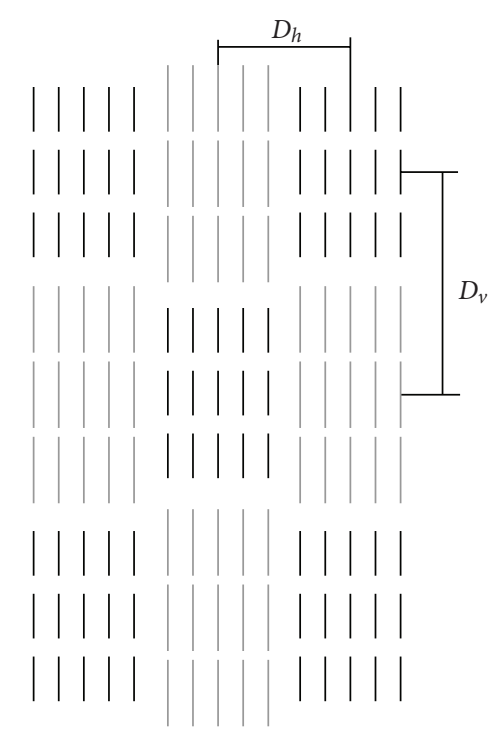

(c)

Figure 1: (a) Schematic representation of the uniform metasurface. (b) Unit cell geometry: top and lateral views. (c) MF superstrate, gray strips resonating at a low resonant frequency and black strips resonating at a high resonant frequency.

coupling coefficients, spherical near-field measurements, and far-field radiation patterns of the array were reported in [9].

In the present paper, a novel multifrequency antenna design using metasurfaces as follows from the previous studies [5-9] is experimentally investigated. The mutual coupling coefficients, when the dipoles are radiating in free space and with the metasurface, are calculated by $S$-parameters measurements and by planar near-field (NF) inspection of the radiated field. The design of the individual antennas was optimized for best impedance matching, maximum gain, and minimum coupling between them in the presence of the metasurface. In the last section, far-field measurements of the radiation pattern with and without the superstrate are presented to prove the benefits of the metasurface.

\section{Metasurface}

The metasurface used in this work as superstrate of the planar antenna is schematically depicted in Figure 1(a). A detailed explanation of the physical phenomena behind this structure can be found in [6]. The metasurface is formed by three layers. The first and third grids consist of parallel short metal strips, while the second one consists of continuous wires. At a certain frequency every pair of strips in grids 1 and 3 (see Figure 1(a)) shows a magnetic resonant mode. The currents induced on individual paired metal strips cancel each other, allowing the incident wave to propagate through the grids. These magnetic dipole moments are local, but every strip pair in the superstrate will radiate principally in a similar manner leading to an approximately uniform aperture phase distribution on the outer superstrate surface. The key idea behind this configuration is that when the metasurface is illuminated by a primary source tuned to the resonant frequency of the metasurface, the radiated field is spread over a larger radiating aperture enhancing its gain.

In order to design an MF metasurface, a combination of two unit cells tuned to two different resonant frequencies in a chessboard-like layout was created. A schematic representation of the metasurface is shown in Figure 1(c). The gray strips are tuned to the so-called Low Resonant Frequency (LRF) and the black ones to the High Resonant Frequency (HRF). The dimensions of both of unit cells are the following.we have LRF: $l_{d}=6.60, l=7.32, t=0.58, D=2.30$, $h=0.5$, and $\varepsilon_{r}=4$; HRF: $l_{d}=5.30, l=6.17, t=0.50, D=2.30$, $h=0.5$, and $\varepsilon_{r}=4$. The distance between centers of cells (HRF to LRF) is $D_{h}=12.71 \mathrm{~mm}$ and $D_{v}=20.95 \mathrm{~mm}$.

To find the transmission window of the LRF and HRF unit cells, the transmission $(T)$ and reflection $(R)$ coefficients were calculated with Ansoft-HFSS under normal incident plane wave excitation. The unit cell excitation is depicted in the inset of Figure 2. Two wave ports were defined on the top and bottom faces of the unit cell and Periodic Boundary Conditions were applied on the sides in order to simulate an infinite slab. The results are presented in Figure 2. Both of unit cells exhibit a transmission window from 9 to $11 \mathrm{GHz}$ in the case of the LRF cell and from 11 to $13.6 \mathrm{GHz}$ in the case of the HRF.

Once the passband of the cells was known, dipole antennas tuned to the transmission window of the cells were designed in order to make an MF dipole antenna array.

\section{Multifrequency Dipole Antenna Array}

In [9], the mutual coupling between dipole antennas with this kind of metasurface as superstrate and the effect in the radiation properties (e.g., radiation pattern, radiation efficiency) was analyzed for a square array formed by $2 \times 2$ 


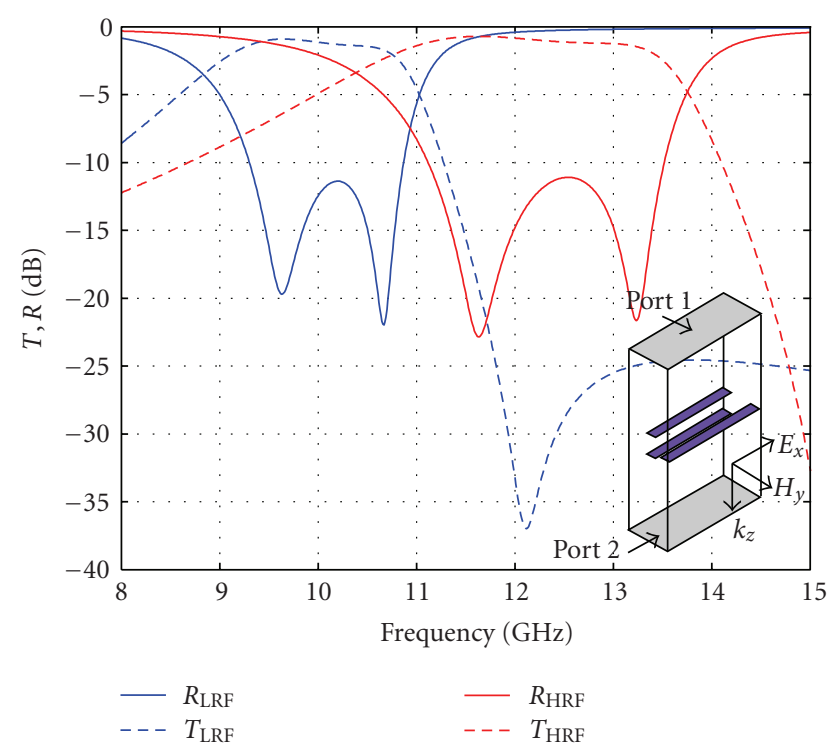

Figure 2: Transmission and reflection coefficients of the LRF and HRF manufactured in FR4 under normal incident plane wave excitation. Inset: unit cell excitation.

dipoles operating at a certain frequency. Two different approaches were used: $S$-parameters measurements, which allow evaluating the coupling coefficients, and near-field measurements of the surface illumination. The radiation coming from the balun was also investigated and it was concluded that in the presence of the superstrate the configuration operates better when the antennas are flipped upside down, so that the baluns are on the bottom face and their ground plane is facing upwards.

A similar analysis to the one carried out for the $2 \times 2$ dipole antenna array has been performed for an MF array. The array is formed by 6 dipoles (see Figure 3(a)) fed by a microstrip-to-coplanar stripline (CPS) balun to match the SMA connector to the CPS [10]. The dipoles in the middle resonate at the LRF and the 4 dipoles at the corners resonate at the HRF. Afterwards, the nonuniform metasurface was placed on top of the array with the dipoles operating at the resonant frequency of the group of cells on top of them (see Figure 3(b)). As it was observed in the singlefrequency array [9], the resonant frequency of the dipoles shifts when the metasurface is placed on top of array. Therefore, LRF and HRF will be used to distinguish between the two type of dipoles, rather than specifying the resonant frequency, since it depends on the presence or absence of the superstrate. The dielectric material used as substrate was FR4. Pictures of the manufactured multifrequency antenna array (MFAA), when the dipoles are radiating in free space and with the superstrate, are shown in Figures 3(a) and 3 (b), respectively. The dipole antennas were designed for good input impedance matching and gain enhancement around the transmission window in the presence of the metasurface, that is, taking into account the loading effect of the superstrate.
3.1. Coupling Coefficients. The S-parameters of the array were measured with a two-port vector network analyzer (Agilent 8722ES) with and without superstrate. In each case, two dipoles were fed and the others were matched with $50 \Omega$ loads to avoid reflections.

The coupling values were obtained by normalizing the measured $S_{21}$-parameter with the radiated power and eliminating the dependence with the impedance matching of the antennas. Therefore, the coupling coefficient $C$ reads

$$
|C|^{2}=\frac{\left|S_{21}\right|^{2}}{\left(1-\left|S_{11}\right|^{2}\right)\left(1-\left|S_{22}\right|^{2}\right)} .
$$

Using the measured $S$-parameters of every two-port combination, the input impedance matching $\left(S_{i i} i=1,2\right)$, $\mathrm{H}$-plane coupling $\left(C_{H}\right)$, E-plane coupling $\left(C_{E}\right)$, and crosscoupling $\left(C_{c}\right)$ (coupling between the top-left and bottomright dipoles) were derived. The results, when the dipoles are radiating in free space (without superstrate) and in the presence of the superstrate are presented in Figure 4. The first column corresponds to the case of dipoles radiating in free space and the second one in the presence of the metasurface. The first row represents the coupling coefficients between HRF dipoles, the second one between LRF dipoles, and the third one cross-frequency coupling between HRF and LRF ones. Due to the geometry of the array, the $\mathrm{E}, \mathrm{H}$, and crosscoupling for the HRF were measured. However, only the Hplane coupling can be measured for the LRF dipoles.

When the fed dipole is radiating in free space, the $\mathrm{H}$ plane coupling with its counterpart dipoles, that is, the ones operating at the same frequency as in Figures 4(a) and $4(c)$, is the most significant, with coupling values around $C_{H}=-20 \mathrm{~dB}$ at the resonant frequency. The cross and $\mathrm{E}-$ plane couplings are around $-35 \mathrm{~dB}$. The input impedance matching of the dipoles is $S_{11}=-13 \mathrm{~dB}$ at $12.15 \mathrm{GHz}$ for the HRF and $S_{22}=-18 \mathrm{~dB}$ at $10.15 \mathrm{GHz}$ for the LRF.

When the metasurface is placed on top of the array (Figures 4(b) and 4(d)), a frequency shift is observed due to the loading effect of the metasurface. The input impedance matching is $S_{11}=-40 \mathrm{~dB}$ at $13 \mathrm{GHz}$ for the HRF dipoles and $S_{22}=-17 \mathrm{~dB}$ at $10.80 \mathrm{GHz}$ for the LRF ones. In the case of the HRF dipoles, a second minimum of the $S_{11}$ is observed around $10.5 \mathrm{GHz}$, which is due to a resonance in the feeding balun. This resonance can be mitigated by surrounding the balun with absorbing material, avoiding the distortion in the radiation pattern. As far as the coupling is concerned, the $\mathrm{H}$-plane coupling decreases to $-40 \mathrm{~dB}$, which means $20 \mathrm{~dB}$ coupling reduction with respect to free space. The $\mathrm{E}$ and cross-coupling remain at a similar level: $C_{c}=-45 \mathrm{~dB}$ and $C_{E}=-35 \mathrm{~dB}$.

Although the dipole antenna was optimized to have good matching, coupling reduction, and gain enhancement in the presence of the superstrate, an input impedance matching better than $-13 \mathrm{~dB}$ was obtained in both cases, with and without the superstrate.

In the case of the cross-frequency coupling, that is, coupling to the LRF dipoles when the HRF one is fed and vice versa (Figures $4(\mathrm{e})$ and $4(\mathrm{f})$ ), the E-plane and cross-coupling values were calculated. When the dipoles are 


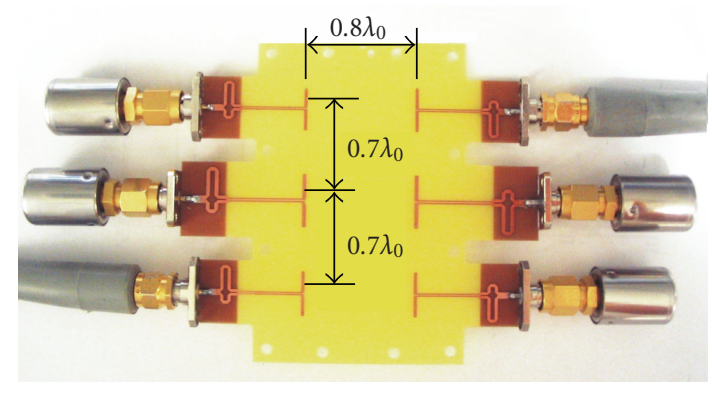

(a)

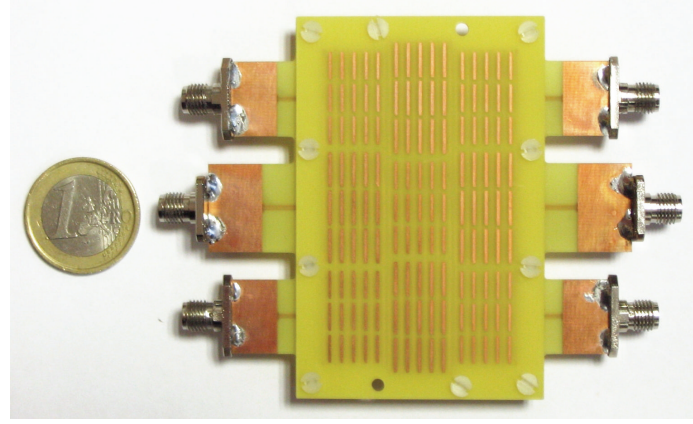

(b)

Figure 3: Multifrequency dipole antenna arrays. (a) Printed dipoles without superstrate. (b) Dipoles with superstrate. Distance between antennas: E-plane distance $=0.7 \lambda_{0} ; \mathrm{H}$-plane distance $=0.8 \lambda_{0}$ at $10 \mathrm{GHz}$.

TABLE 1: E- and H-plane coupling (in $\mathrm{dB}$ ) at the resonant frequency.

\begin{tabular}{|c|c|c|c|}
\hline & HRF & LRF & Cross-freq. $f_{12}$ \\
\hline & $C_{H}=-20$ & $C_{H}=-20$ & \\
\hline \multirow[t]{3}{*}{ Air } & $C_{c}=-33$ & & $C_{c \mid \mathrm{HRF}}=-25 ; C_{c \mid \mathrm{LRF}}=-25$ \\
\hline & $C_{E}=-40$ & & $C_{E \mid \mathrm{HRF}}=-29 ; C_{E \mid \mathrm{LRF}}=-25$ \\
\hline & $C_{H}=-40$ & $C_{H}=-40$ & \\
\hline \multirow[t]{2}{*}{ Superstrate } & $C_{c}=-45$ & & $C_{c \mid \mathrm{HRF}}=-43 ; C_{c \mid \mathrm{LRF}}=-44$ \\
\hline & $C_{E}=-33$ & & $C_{E \mid \mathrm{HRF}}=-40 ; C_{E \mid \mathrm{LRF}}=-27$ \\
\hline
\end{tabular}

radiating in free space (Figure $4(\mathrm{e})$ ), the E-plane and crosscoupling are around $-25 \mathrm{~dB}$. However, in the presence of the superstrate (Figure 4(e)), the cross-frequency coupling coefficients decrease to $-40 \mathrm{~dB}$, except for the E-coupling at LRF that remains around $-27 \mathrm{~dB}$. These results are summarized in Table 1.

3.2. Near-Field Measurements. The mutual coupling phenomenon was also investigated by NF measurements of the array. One of the dipoles was fed and the others were matched with $50 \Omega$ loads. By this measurement, the surface illumination and the field that reaches the neighboring dipoles were detected. The frequencies for the near-field measurement were the resonant frequency of the configurations with and without superstrate, respectively. Although the operational frequency is different, the aim of this analysis is to compare two configurations operating at their optimum frequency, that is, at the resonance. Moreover, the variation with frequency of the coupling coefficients in air is very small, as shown in Figure 4 . The vector network analyzer HP8510C provided the microwave signal to feed the antenna and measured the field detected by the probe. A custom LabView program coordinates the motion of the scan with the data acquisition of the VNA.

A monopole antenna was used as a probe. It was mounted over a $2 \mathrm{D}$ scanning system and placed $\lambda_{0} / 4$ away from the antenna. The distance from the antenna to the probe, $z_{0}$, was chosen so that the probe was close enough to measure the surface illumination but remains out of the reactive near field of the antenna, which is usually taken as $\lambda_{0} / 2 \pi[11]$. The step of the acquisition points $\Delta_{x}$ and $\Delta_{y}$ was fixed to $2 \mathrm{~mm}$ so that the sampling criteria $[11,12]$

$$
\Delta_{x}, \Delta_{y}=\frac{\lambda_{0}}{2 \sqrt{1+\left(\lambda_{0} / z_{0}\right)^{2}}}
$$

for measurement distances $z_{0}$ smaller than $\lambda_{0}$ was satisfied for the HRF and LRF. Since the E field radiated by the antenna is polarized along the metal strips, the probe was oriented parallel to the strips and only the copolar polarization was taken into account.

The results of the scanned field when the HRF and LRF dipoles were radiating are shown in Figure 5. When the metasurface is placed atop the array (Figures 5(b) and $5(\mathrm{~d}))$, the power radiated by the driven dipole is confined by the metasurface area that resonates at the same frequency and radiates in the boresight direction, reducing the endfire radiation and, therefore, the mutual coupling between elements. As expected from the $S$-parameters measurements, a clear reduction in $\mathrm{H}$-plane coupling is observed. The E-plane, cross-coupling, and cross-frequency coupling also decrease, showing overall a good level of isolation between antennas.

3.3. Radiation Pattern. In order to check the effect of the mutual coupling on the radiation performance of the antenna, the radiated far field was measured in the DTUESA Antenna Test Facility with and without the metasurface at the resonant frequency. Only one dipole was fed and the others were matched with $50 \Omega$ loads. The normalized measurements of the E- and H-plane cuts of the radiation 


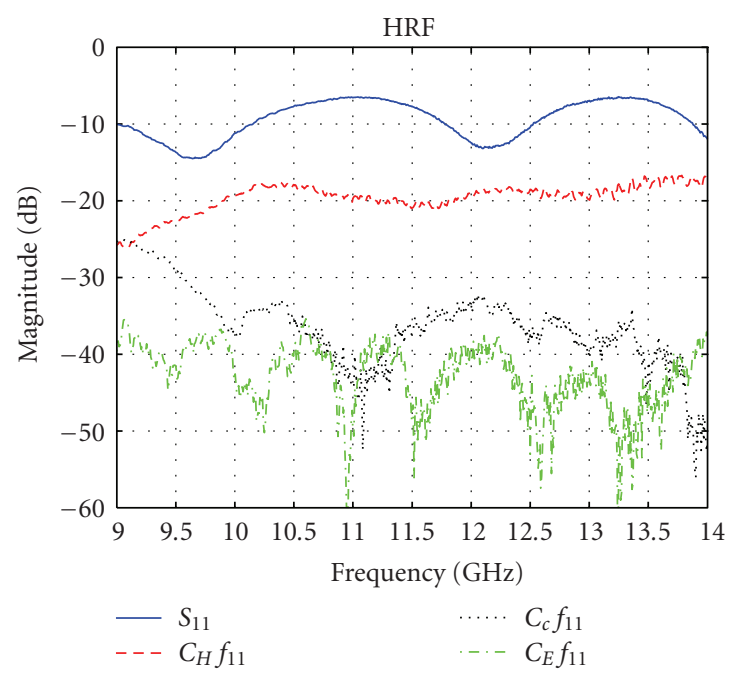

(a)

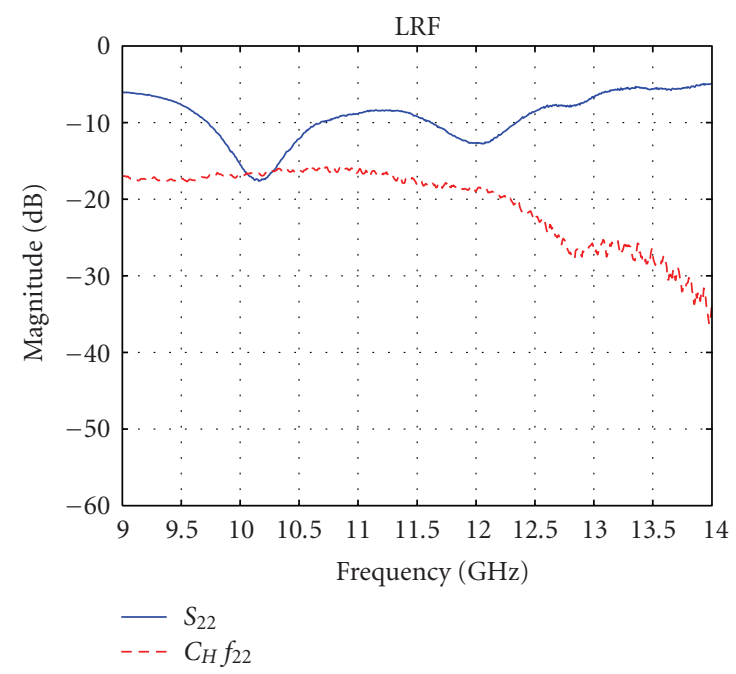

(c)

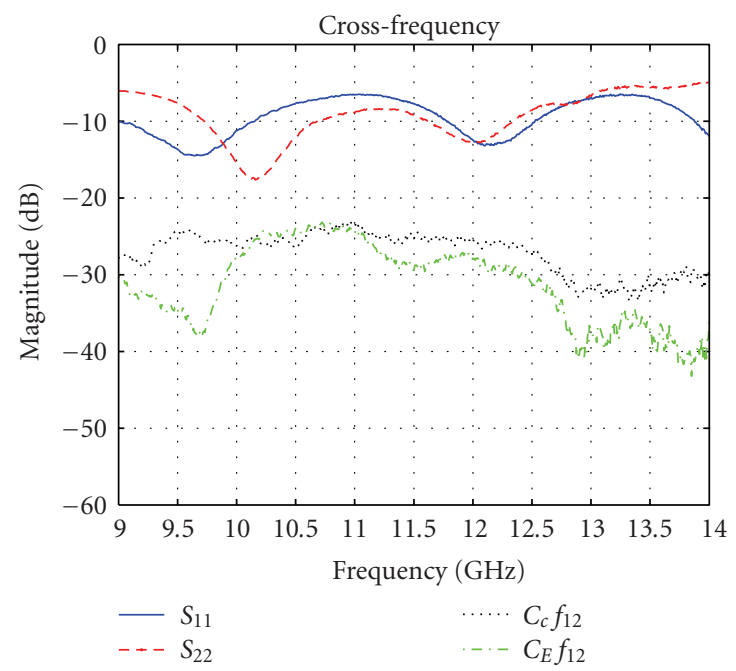

(e)

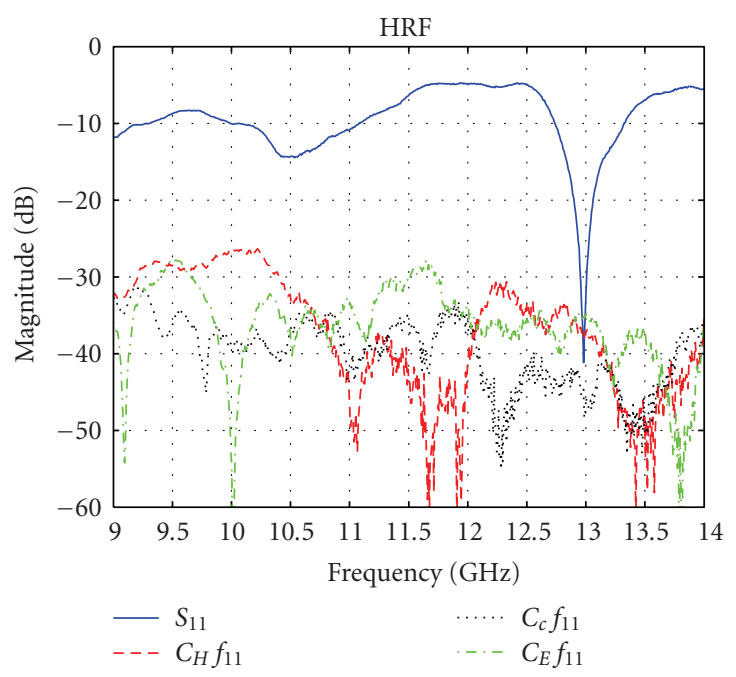

(b)

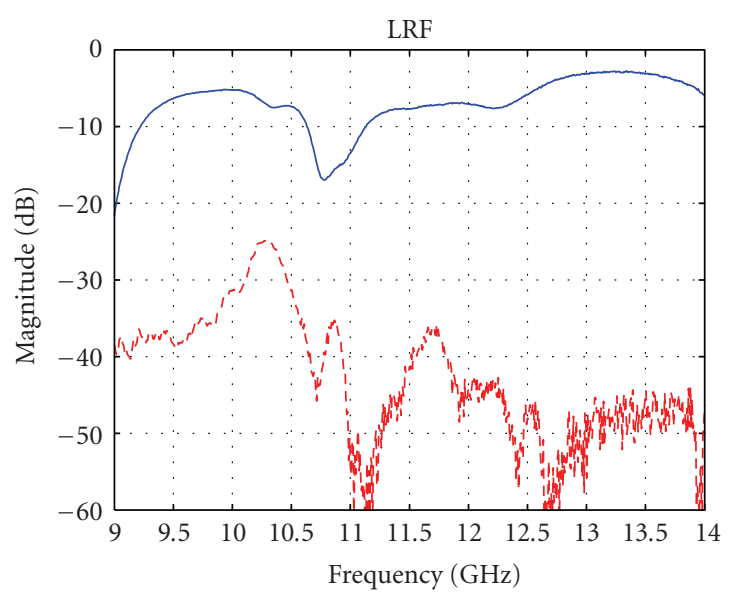

$$
-S_{22}
$$$$
\text { -- } C_{H} f_{22}
$$

(d)

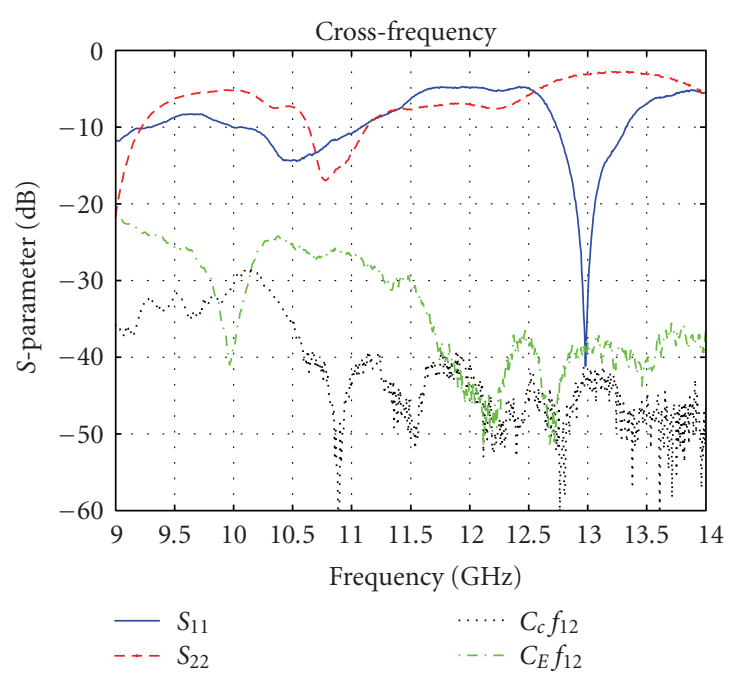

(f)

Figure 4: Coupling coefficients calculated from the $S$-parameters measurements: Left column without superstrate (free space) and right column with superstrate. (a), (b) Coupling between dipoles operating at $f_{1}=$ HRF. (c), (d) Coupling between dipoles operating at $f_{2}=$ LRF. (e), (f) Coupling between dipoles operating at different frequencies $f_{12}$ (cross-frequency coupling). 


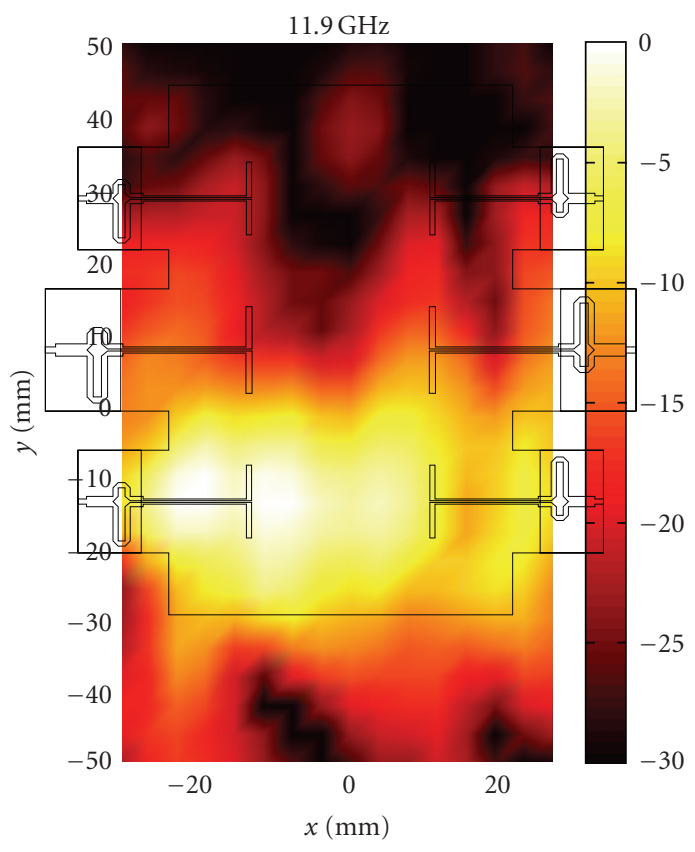

(a)

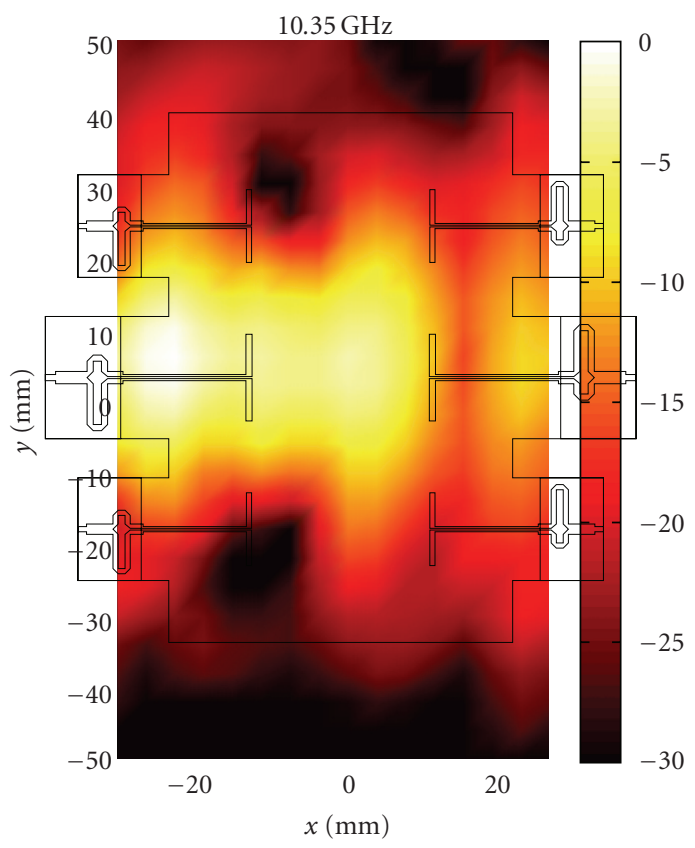

(c)

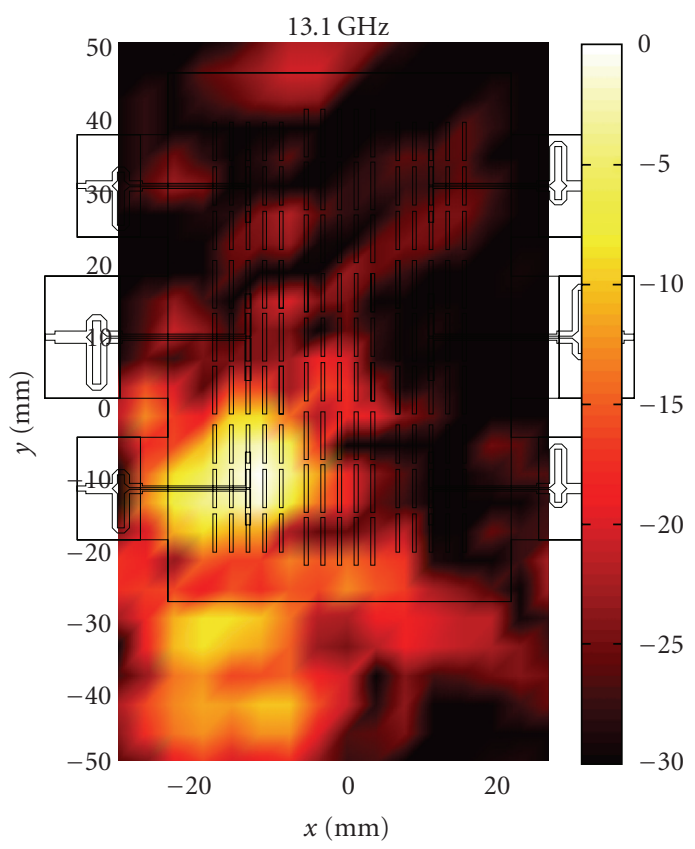

(b)

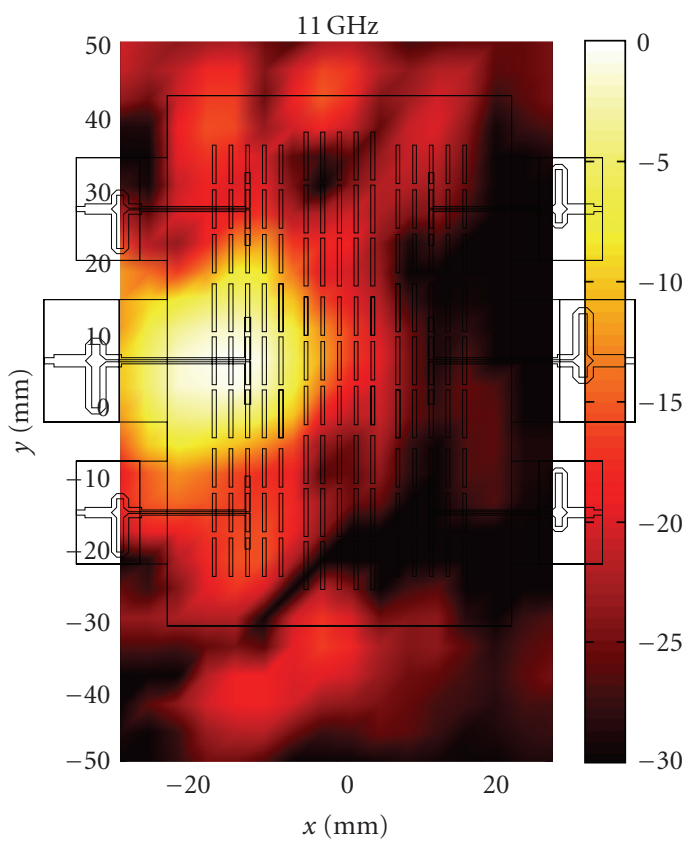

(d)

Figure 5: Near-field measurements of the surface illumination when the dipoles resonating are radiating in free space(left column) and with the superstrate (right column). (a), (b) Resonant frequency at $f_{\mathrm{HRF}}$. (c), (d) Resonant frequency at $f_{\mathrm{LRF}}$.

pattern in the absence and presence of the metasurface for the HRF and LRF dipoles are shown in Figure 6. The radiation is along the boresight direction.

When the HRF or LRF dipoles are radiating in free space at the resonant frequency (Figure 6(a)), due to the strong $\mathrm{H}$ plane coupling between the elements and the presence of the ground plane in this plane, the radiation pattern is strongly distorted (dashed line). Instead of a uniform circular shape, several peaks and nulls appear. The low coupling measured in the E-plane is confirmed with the radiation pattern; very low radiation is observed in the endfire direction and the radiation pattern is almost symmetrical in both of half spaces.

When the superstrate is placed on top of the array (Figure 6(b)), the power radiated by the antenna, is picked up by the superstrate, confined over a larger area on top of the antenna and reradiated producing an enhancement of the boresight radiation and a reduction of the back radiation. 


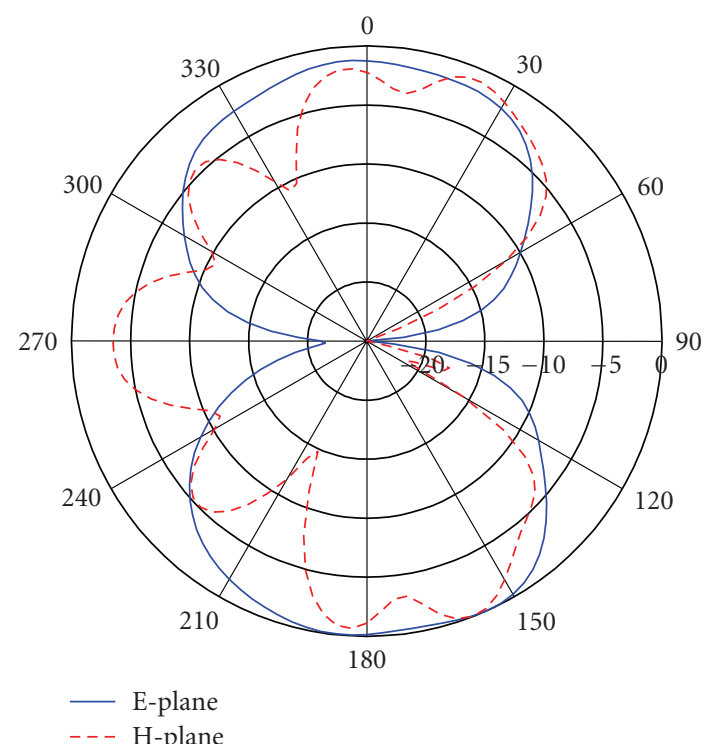

(a)

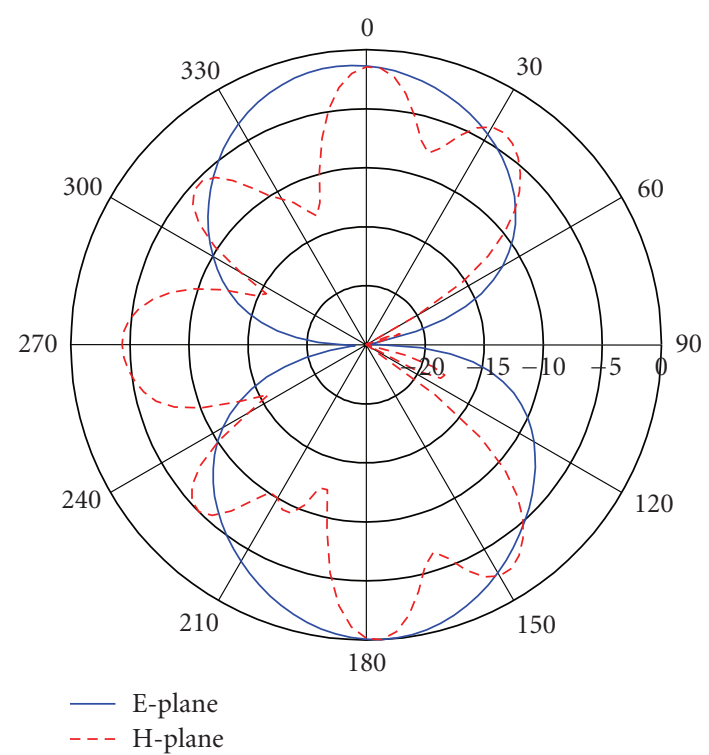

(c)

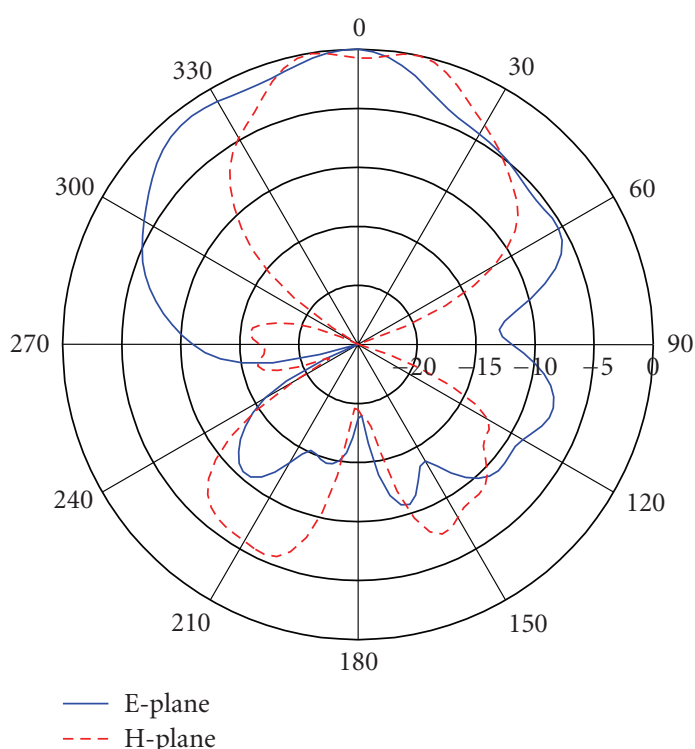

(b)

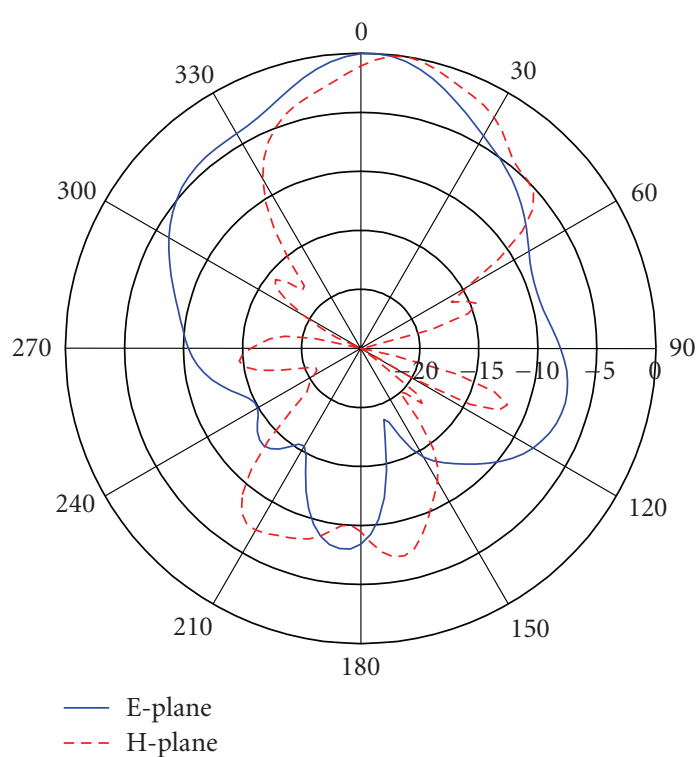

(d)

FIGURE 6: Normalized E- and H-plane cuts of the radiation pattern. (a) HRF dipole without superstrate at $12 \mathrm{GHz}$. (b) HRF dipole with superstrate at $13.1 \mathrm{GHz}$. (c) LRF dipole without superstrate at $10.3 \mathrm{GHz}$. (d) LRF dipole with superstrate at $11 \mathrm{GHz}$.

Due to the power confinement and the H-plane coupling reduction, the $\mathrm{H}$-plane endfire radiation is strongly reduced. Notice that although the E-plane coupling increases in the presence of the superstrate, the E-plane cut of the radiation pattern is not distorted in the boresight direction, but only an increase of the E-plane endfire radiation is observed.

\section{Conclusions}

In the present paper, the mutual coupling between dipoles in an MFAA was experimentally investigated. A metasurface was used as superstrate in order to reduce the coupling between elements of the array. The reduction in mutual coupling was demonstrated by $S$-parameters and near-field measurements of the radiated field. In the case of the $\mathrm{H}$ plane coupling, $20 \mathrm{~dB}$ coupling reduction was observed due to the presence of the superstrate. Although the E-plane coupling increases with respect to the free-space case due to the guiding of the fields along the strips, it remains below $-20 \mathrm{~dB}$.

By near-field scanning measurements, it was observed that, due to the resonant behavior of the metasurface, the power radiated by the dipoles is confined and directed to the boresight. This reduces the endfire radiation and, therefore, the coupling with the surrounding dipoles. In addition, this provides a way of isolating individual dipoles from their 
respective neighbors, which is required for imaging array applications. Apart from the MF capability, polarization distinction could be added to the array by rotating one of the sets of dipoles (HRF or LRF) $90^{\circ}$. This would increase the capacity and compactness of the array and its performance for imaging array applications.

\section{Acknowledgments}

This research has been financially supported by METAMORPHOSE NoE funded by E. C. under contract no. NMP3CT-2004-50252 and Spanish Government under project no. TEC2006-13248-C04-03/TCM. One of the authors (K. Guven) acknowledges support of TUBITAK under the Project no. 106E198. The authors would also like to thank Professor O. Brenjerg and Dr. S. Pivnenko from DTU for the assistance with the radiation pattern measurements.

\section{References}

[1] T.-H. Chu and N. H. Farhat, "Frequency-swept microwave imaging of dielectric objects," IEEE Transactions on Microwave Theory and Techniques, vol. 36, no. 3, pp. 489-493, 1988.

[2] V. Semenchik, V. Pahomov, and S. Kurilo, "Multifrequency microwave imaging," in Proceedings of the 5th International Kharkov Symposium on Physics and Engineering of Microwaves, Millimeter, and Submillimeter Waves (MSMW'04), vol. 1, pp. 193-195, Kharkiv, Ukraine, June 2004.

[3] R. Gonzalo, P. de Maagt, and M. Sorolla, "Enhanced patch-antenna performance by suppressing surface waves using photonic-bandgap substrates," IEEE Transactions on Microwave Theory and Techniques, vol. 47, no. 11, pp. 21312138, 1999.

[4] I. Ederra, L. Azcona, B. E. J. Alderman, et al., "A $250 \mathrm{GHz}$ subharmonic mixer design using EBG technology," IEEE Transactions on Antennas and Propagation, vol. 55, no. 11, part 1, pp. 2974-2982, 2007.

[5] E. Saenz, R. Gonzalo, I. Ederra, J. C. Vardaxoglou, and P. de Maagt, "Resonant meta-surface superstrate for single and multifrequency dipole antenna arrays," IEEE Transactions on Antennas and Propagation, vol. 56, no. 4, pp. 951-960, 2008.

[6] P. M. T. Ikonen, E. Saenz, R. Gonzalo, and S. A. Tretyakov, "Modeling and analysis of composite antenna superstrates consisting on grids of loaded wires," IEEE Transactions on Antennas and Propagation, vol. 55, no. 10, pp. 2692-2700, 2007.

[7] E. Saenz, I. Ederra, P. De Maagt, and R. Gonzalo, "Highly efficient dipole antenna with planar meta-surface," Electronics Letters, vol. 43, no. 16, pp. 850-851, 2007.

[8] E. Saenz, K. Guven, I. Ederra, E. Ozbay, P. De Maagt, and R. Gonzalo, "Near-field measurement of a planar metasurface illuminated by dipole antennas," in Proceedings of the Loughborough Antennas and Propagation Conference (LAPC '08), pp. 221-224, Loughborough, UK, March 2008.

[9] E. Saenz, I. Ederra, R. Gonzalo, S. Pivnenko, O. Breinbjerg, and P. de Maagt, "Coupling reduction between dipole antenna elements by using a planar meta-surface," IEEE Transactions on Antennas and Propagation, vol. 57, no. 2, pp. 383-394, 2009.

[10] Y. Qian, W. R. Deal, N. Kaneda, and T. Itoh, "Microstrip-fed quasi-Yagi antenna with broadband characteristics," Electronics Letters, vol. 34, no. 23, pp. 2194-2196, 1998.
[11] A. Yaghjian, "An overview of near-field antenna measurements," IEEE Transactions on Antennas and Propagation, vol. 34, no. 1, pp. 30-45, 1986.

[12] Y. T. Looand and S. W. Lee, in Antenna Handbook, J. AppelHansen, Ed., Van Nostrand Reinhold, New York, NY, USA, 1998. 

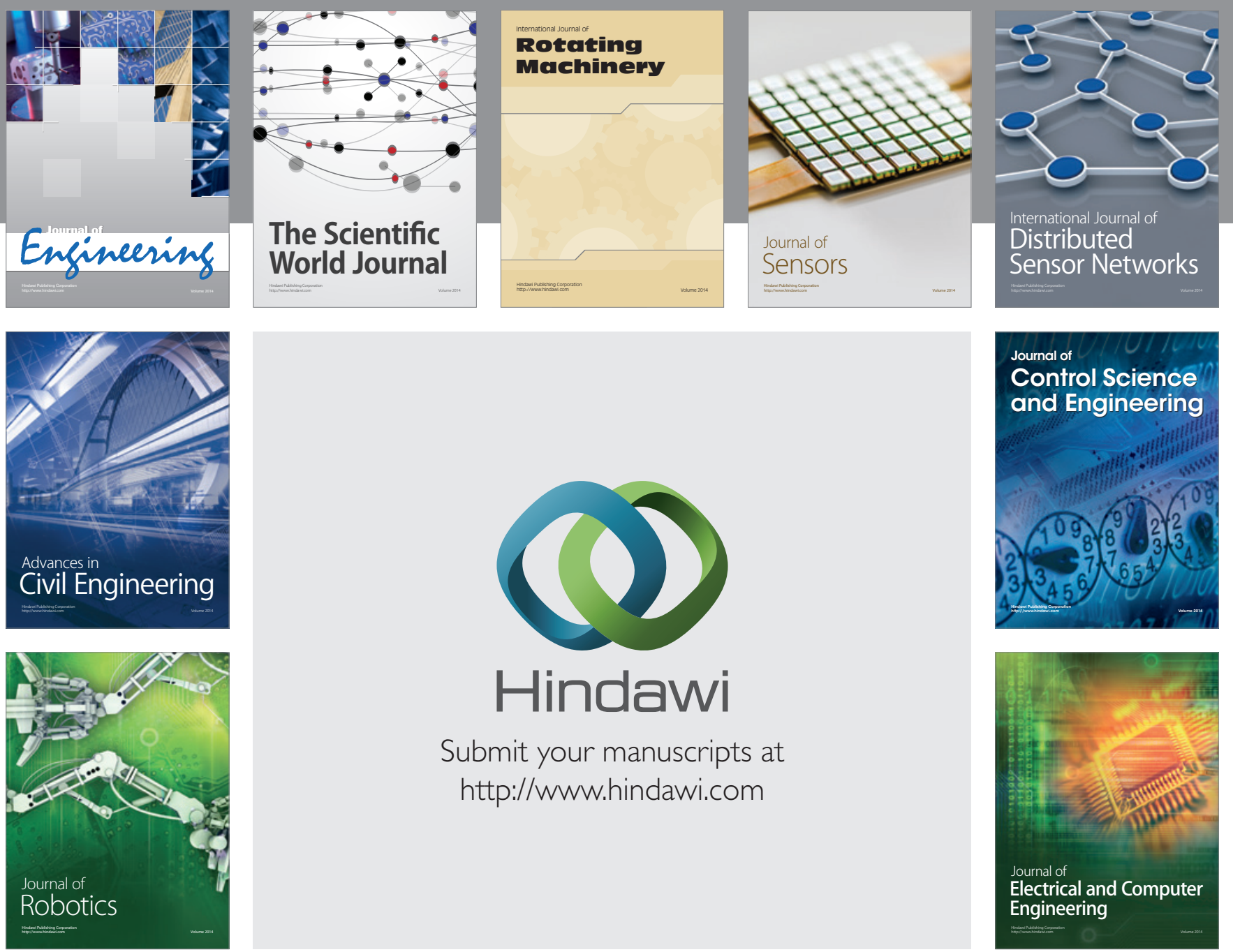

Submit your manuscripts at

http://www.hindawi.com
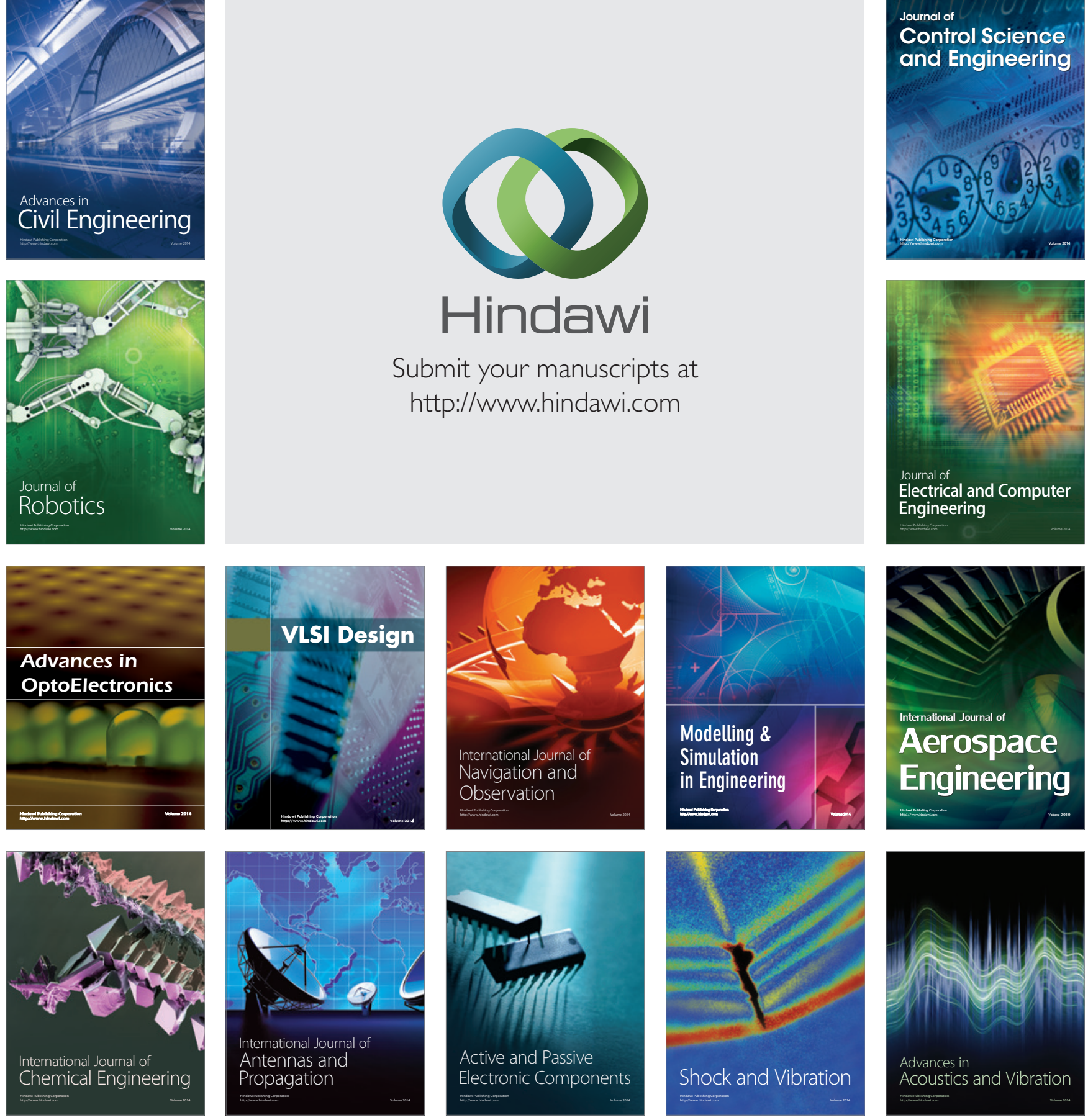